\title{
Reverse distal femoral locking plate for subtrochanter femur fracture
}

\author{
Peter Giarso, Ismail H. Dilogo
}

Department of Orthopaedic and Traumatology, Faculty of Medicine, Universitas Indonesia, Cipto Mangunkusumo Hospital, Jakarta, Indonesia

\section{ABSTRACT}

In these case series, we used titanium locking compression plate-distal femur (LCP-DF) plate (Synthes) 9-11 hole using less invasive stabilization system or open reduction technique. This case series aims to determine the functional scores on reverse distal femoral locking plate for subtrochanteric femur fracture. A34-year-old male with closed subtrochanteric fracture of the right femur (Seinsheimer 2B) with Harris hip scores (HHS) of 17, 96, and 97 obtained consecutively in 0, 6, and 12 months, respectively. A 24-year-old male with closed comminuted subtrochanteric fracture of the right femur (Seinsheimer V) with HHS of 13, 93, and 97 at 0, 6, and 12 months respectively. A 39-year-old male with non-union, left subtrochanteric femur fracture (Seinsheimer 2C) yielded HHS of 38, 73, and 77 at 0, 6, and 12 months, respectively. A 35-year-old female with close subtrochanteric fracture of the right femur (Seinsheimer IIB) yielded HHS of 23, 40, and 73 at 0, 6, and 12 months, respectively. Mean initial HHS and scores at 6 and 12 months reached 22,75 , and 86 , respectively.

Keywords: subtrochanteric fracture, reverse distal femoral locking plate

pISSN: 0853-1773• eISSN: 2252-8083• https://doi.org/10.13181/mji.v27i2.1377• Med J Indones. 2018;27:121-7

- Received 09 Feb 2016 • Accepted 20 Apr 2018 
Subtrochanteric fracture remains one of the most prevalent fracture case and also one of the current public health issue, presenting difficulty in management and unsatisfactory output. ${ }^{1}$ Internal fixation is the treatment of choice for this type of fracture. Various types of implants are made from intramedullary implants and extramedullary plates and screws. Various extramedullary implants include the sliding hip screw, dynamic condylar screw, angled blade plate, and proximal femoral plate. ${ }^{2}$ Although intramedullary implant is an option for unstable fractures, not all cases can use this type of implant, for example, cases with fracture lines reaching the piriformis fossa (Russel Taylor II), patients with short-bone and narrow medulla, and teens with having a feature of the epiphyseal plate deformity and severe deformity of the femur. ${ }^{3,4}$ Extramedullary implant is an option.

Furthermore, many reports claimed that $40 \%$ of patients with intramedullary implants complained about peritrochanteric pain, stiffness, and limping. The hip abductor components exert a significant influence. An extremely lateral entry point can cause damage to the tendons of the gluteus medius by up to $25 \%$. Superior gluteal nerve injury can occur during reaming of the piriformis fossa, especially during hip flexion and adduction. Vascular disorders can also occur related to the holes in the piriformis fossa. These events are absent in extramedullary implants. ${ }^{5-7}$

Dynamic compression plate-distal femoral (DCP-DF) (Synthes $®$ ) is designed for the treatment of distal femur fractures using the principle of internal splinting with minimally invasive techniques (less invasive stabilization system (LISS)/LISS-DF). In biomechanics, compared with the non-locking plate, conventional plates require bone compression and a bone-plate friction surface. Repeated axial power causes a loosening of the screws and plate. In the early aspect, a repetitive force causes an instability especially in the metaphyiscal and osteoporotic bone. In addition to bone compression, a periosteum damage also occurs. ${ }^{8}$ Titanium is theoretically superior in terms of fatigue resistance and modulus of elasticity than stainless steel compared to other fracture types; the use of compression plates for distal femoral fracture is the most widely reported case with the most satisfactory results. Most reports indicate a satisfactory union of figures and low complications, such as implant failure or varus deformity. ${ }^{9}$
The use of plate DCP-DF for proximal femoral fractures has been reported with satisfactory results. This broader indication is by using contralateral implants, by reverse the plate and mounted on the proximal femur. Sidhom et $\mathrm{al}^{10}$, used this plate for patients with subtrochanter fractures, by flipping the plate and support jig with the incision was not necessarily wide and reduced the fracture easily.

Ma et $\mathrm{al}^{11}$ reported a case series of 20 patients with unstable trochanteric fractures, which were successfully managed via the DCPDF with an average operating time of 60 minutes, bleeding volume of $150 \mathrm{ml}$, and $5.5 \mathrm{~cm}$ incision. Based on the clinical outputs (pain and range of movement), functional, radiological (neck shaft angle and a sign of union), and the incidence of complications, the results showed no difference with the use of other implants based on literature. No screw pull-out was observed with $100 \%$ union figure (11 patients featured delayed union in six months, but continued follow-up presented signs of union without extra management). Two cases included a porotic case with a broken screw and early weight bearing. The broken screw closest to the fracture line on the bicortical may be too rigid and cannot resist motion. At the medial cortical comminuted fracture, implants are attached to the fracture line to obtain the highest loads. Broken screw causes varus and limb length discrepancy. This study recommends the reverse DCP-DF implant as an alternative.

Acklin et $\mathrm{al}^{12}$ reported 14 cases with an average age of 52 years. An average operating time of $98 \mathrm{~min}$ is required with 164 of fluoroscopy. Ten cases were cured under the mean operating time, with two cases of broken screws on the bicortical side near the fracture line. Broken screw occurs in cases with very proximal fracture line. Acklin also recommends DCP-DF as an alternative for trochanteric fractures.

Zhang et $\mathrm{al}^{8}$ also reported the use of reverse DCP-DF for 28 cases of stable or unstable intertrochanteric fracture in old-age patients (median 82.3 years). Although it is not rated as functional output, the technical operations LISS for reverse plate are easy for the operator (the same as for the installation of the distal femur) and provide good results for radiological control (100\% union within six months). 
In the case of periprosthetic fracture, Ehlinger et $\mathrm{al}^{13}$ reported that the use of DCPDF plate could increase fixation because of bicortical locking screw and the number of screws that can be mounted on the proximal part to hold the trochanter in case of proximal periprosthetic fracture extensions. Plate length benefits from the type of fracture extensions to the distal femur. The limitation is in the shape of the headplate on the sagittal plane is less curved (because it is made in accordance with the sagittal plane of the condyle of the distal femur). The aim of this case series is to evaluate the use of the reverse distal femoral locking plate for subtrochanter femur fracture.

\section{CASE REPORT}

\section{Case 1}

The case involved a 34-year-old male patient who suffered from traffic accidents. The patient was diagnosed with polytrauma, floating hip with martin taylor classification (MTC) 1 pelvic fractures; fractures were also observed in the tibial plafond (ipsilateral) and the shaft of the humerus (Figure 1). Subtrochanteric ipsilateral fracture was identified with Seinsheimer 2B classification. Damage control was achieved with the installation of skeletal traction in the emergency department. Definitive surgery was performed after 10 days.

All fractures patient were treated with operative therapy. For subtrochanteric fracture, a surgery with open reduction and implant reverse plate in the distal femur was performed using LCP-DF 4.4/5.0 9 left holes to the right femur fracture. As for the other fractures, an open reduction and implant placement were also performed. The distal plate femur mounted in the reverse contralateral and the proximal locking screw was also filled in the distal fragment with four mounted screws. The screws were mounted at the interfragmen fracture site. Post-operative roentgen, the fracture line was observed, and no varus or valgus deformity was noted. The screws in the bones failed to penetrate the joints, along with placement of the tip of the right plate.

As multiple fracture cases need to be operated gradually, the patient stayed for more than two weeks in the hospital. The patient was immobilized for a long period. No complications were observed during and after the surgery. Passive motion exercises were conducted since from the hospital. The patient performed walking exercise using a cane for the axial load.

Follow-up was performed in 28 weeks, and bone healing was evident according to the radiological description (Figure 1). The proximal and distal fragments fell in one line, and neither varus nor valgus deformity was detected. No implant failure, such as broken or pull out, was observed. Clinically, the patient was able to walk with a full load without using a cane. Hip and thigh pain scored $0-1$ in the visual analog scale. Harris hip score (HHS) was 96. No difference was observed in the anatomical length of the femur.

\section{Case 2}

A 24-year-old male patient with multiple trauma due to traffic accidents was admitted to the emergency room with hypovolemic shock. Figure 2 showed radiological results were obtained from
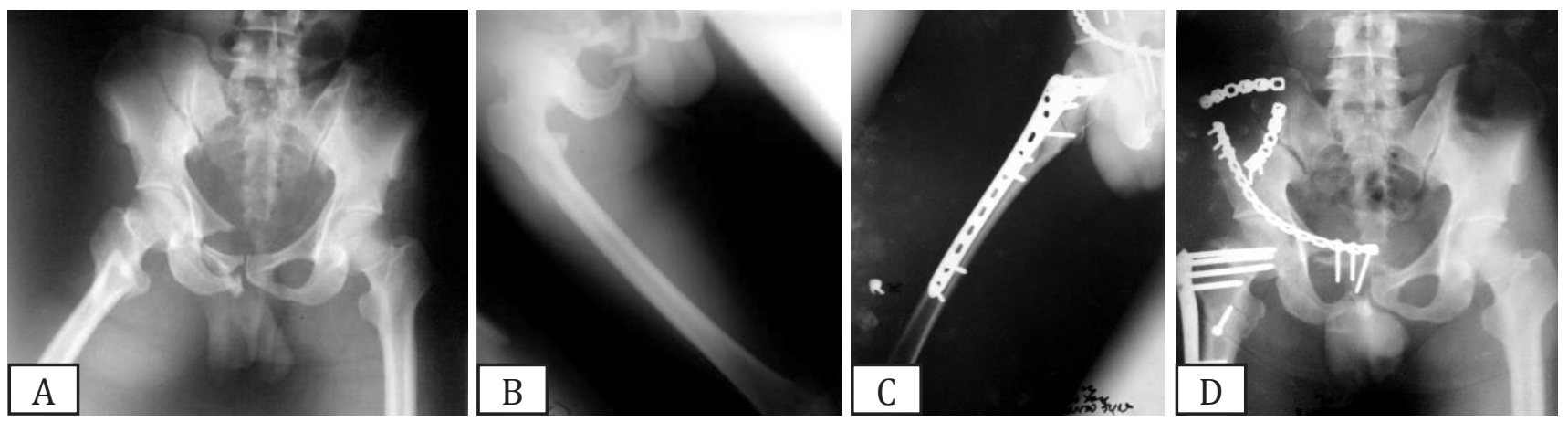

Figure 1. $(A, B)$ Case 1. Roentgen of proximal femur presented subtrochanter fractures with floating hip; (C,D) Follow-up of 28 weeks. Overview of the fracture line healing. Implants are not shifted nor broken. Bone deformity (varus or valgus) is absent 
MTC 1 pelvic fracture with subtrochanteric femur fracture (Seinsheimer V) (floating hip). The patients also presented grade IV of kidney trauma and ipsilateral fracture of the fibula head. In the emergency room, damage control was achieved by mounting a pelvic anterior frame.

After receiving immunosuppressants for 23 days, the patient underwent a definitive surgery with an open reduction for plate mounting in the pelvis and installation of the reverse LCP-DF for subtrochanteric fractures with LISS technique (Figure 2). After the stabilization of the acetabulum and pelvis, a femur traction was performed, followed by lateral incision on the proximal femur. Five screws can be mounted with a head plate. Although the fracture line extended proximally, the principle of the bridge and internal fixation can be achieved by using a long plate.

After surgery, patient was rehabilitated with an axillary stick without weight-bearing activity. The wound and soft tissues healed completely. The patient yielded HHS of 13, 93, and 97 at 0,6 , and 12 months, respectively, whereas

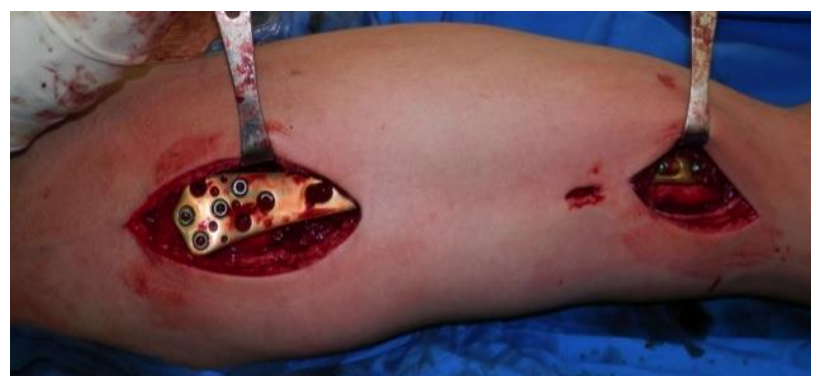

Figure 2. Intraoperative mounting of the plate using minimally invasive techniques
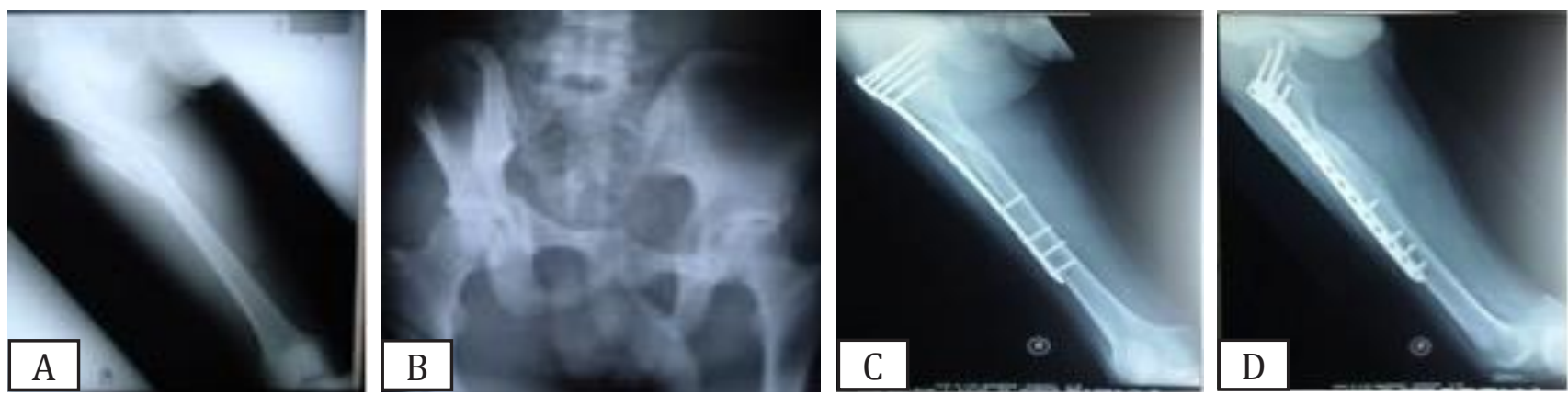

Figure 3. (A,B) Case 2. Roentgen of proximal femur fractures subtrochanter with floating hip; (C,D) X-rays of the femur six months post-surgery radiological connection was achieved at six months (Figure 3). The clinical condition of patient 6 months post-surgery is shown in figure 4.

\section{Case 3}

This case involved a 39-year-old male patient with a history of recurrent fractures of the subtrochanteric region six months prior to the hospital (a neglected case). Previously, the patient experienced a seven-year fracture before hospital admission and an implant surgery. However, the patient still felt pain accompanied with a discharge from the surgical wound (possibly infected non-union). The patient presented a 6 $\mathrm{cm}$ difference in anatomical femur length.

Given the differences in limb length, skeletal traction mounted with weights was aimed at muscle relaxation. Open reduction surgery was performed to perform recanalization and decortication, followed by a cancellous bone graft from the iliac. The 9-hole reverse LCP-DF plate was used. Anatomical femur length was reached clinically and radiologically. However, the patient never came back for control after the operation (Figure 5).

\section{Case 4}

A 35-year-old woman fell down in the bathroom with both legs in stretched position. The right thigh knocked on the bathroom steps. After the incident, the patient complained about severe pain and was unable to walk. She complained about thigh pain, and instability and deformity of limb length $(2 \mathrm{~cm})$ were also observed.

From pre-operative $\mathrm{x}$-rays, the mounting plate on the proximal femur appeared normal and showed no signs of callus formation. The femur 

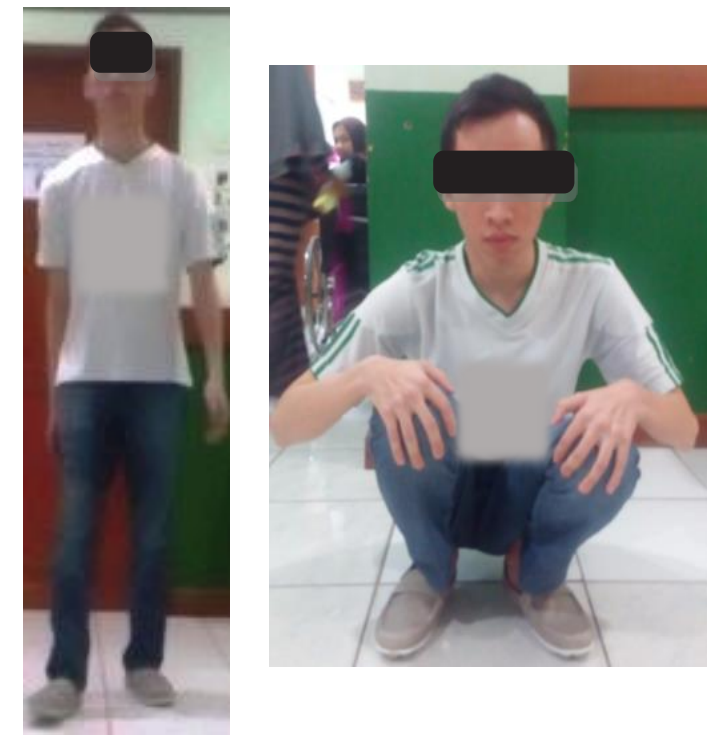

Figure 4. Clinical patient 6 months post-surgery in case 3

head remained vital. The patient was diagnosed with non-union fractures accompanied by deformity. The patient had a diabetes mellitus.

The plate of the patient was replaced with internal fixation by LCP-DF with an additional bone graft. As the surgery will be performed without the implant, it was conducted openly. Good bone quality was observed with a slightly atrophic non-union and instability of the plate. Several screws on the proximal femur were broken. Plate mounting was performed in reverse, and five pieces of screw can be mounted in the proximal femur (Figure 6). The patients can follow the physiotherapy program and walk using a stick. HHS at early stage and at six months reached 40 and 73 , respectively.

\section{DISCUSSION}

Subtrochanteric and proximal femur fractures often occur either due to severe trauma, such as traffic accidents or pathologic trauma. With an assortment of classification and treatment of choice suggesting that this type of fracture is either stable or unstable, no therapy is superior owing to the variations in the form of fracture that may occur. Until now, the trochanteric fracture region remains a challenge for operators owing to the level of difficulty, variations in management, and completeness of instruments.
Although various reports on the intramedullary nail implants showed a satisfactory result in the surgical procedure, the soonest possible time is early mobility and late of output functions, especially in the intertrochanter; not all types of fractures can be handled by these implants. ${ }^{14}$ Extramedullary implants, including plates, remain an option. Various reports compared the use of these implants in terms of their advantages and disadvantages.

Distal femoral plate has also been used as an alternative used for the proximal femur fracture. The platform head with numerous screw holes determines the typical sagittal and coronal arches, the strength of the neck plate, the length and number of holes in the distal plate, titanium plate material (from multiple manufacturers), the principle of internal splint biomechanics and biological advantages (causes no damage in the soft tissue periosteum), and surgery techniques with LISS. The LCP-DF plate that has been used in pathological fractures was reported have failed with conventional management. Then, the use of the LCP-DF plate was expanded and reported for the cases of peritrochanteric fracture and failed implants, whereas recent reports focused at the analytical level and compared the choices of other implant types and the types of fracture.

The LCP-DF serves as an alternative because of the variety and complexity of each case. Occasionally, the LCP-DF is considered as a last resort owing to the type of fracture that prevents another implant from being used. Therefore, these cases not only include the simple cases with isolated fracture of the proximal femur but also those with a concomitant injury, including the general state and those with complex background. Thus, any discussion of a case and its output can be a topic of their own.

The LCP-DF plate on the reported cases is an alternative option when intramedullary implant is especially unusable. The LISS technique was selected for preservation of biological components, although several cases, such as the cases of neglected fracture fragments, involved open reduction surgery.

All the cases are complex cases; patients presented multiple trauma or a neglected condition. Handling started with addressing 

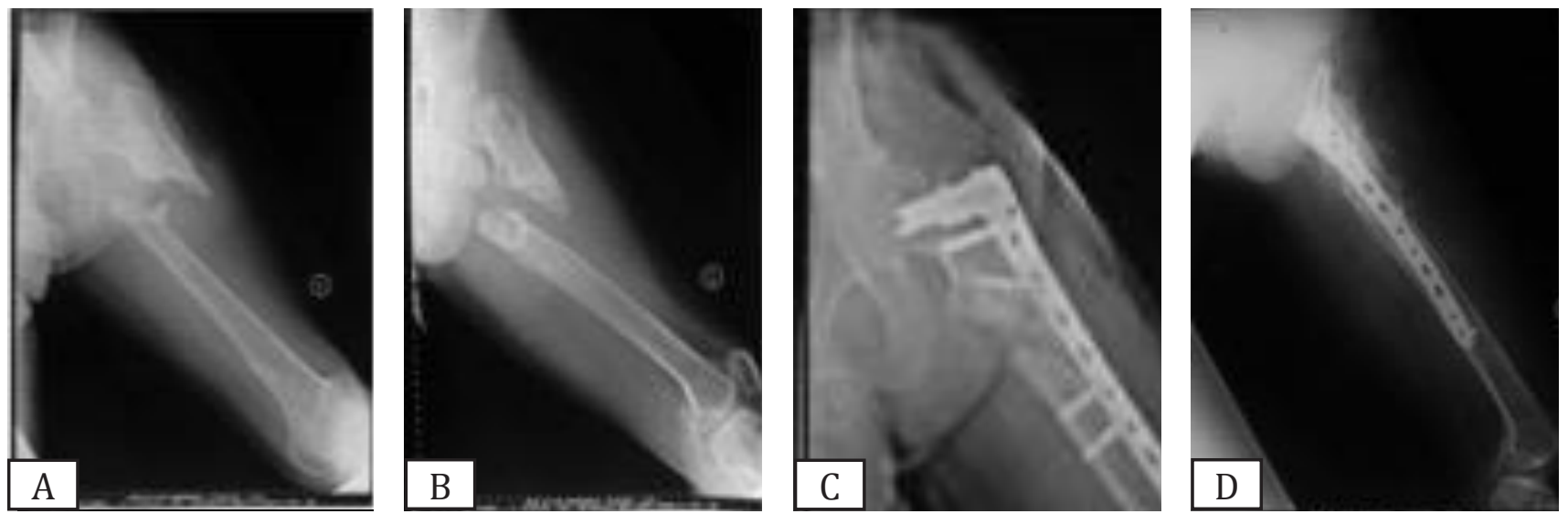

Figure 5. (A,B) Case 3. X-rays of the neglected proximal femur fracture subtrochanter; (C,D) X-rays of post-operative
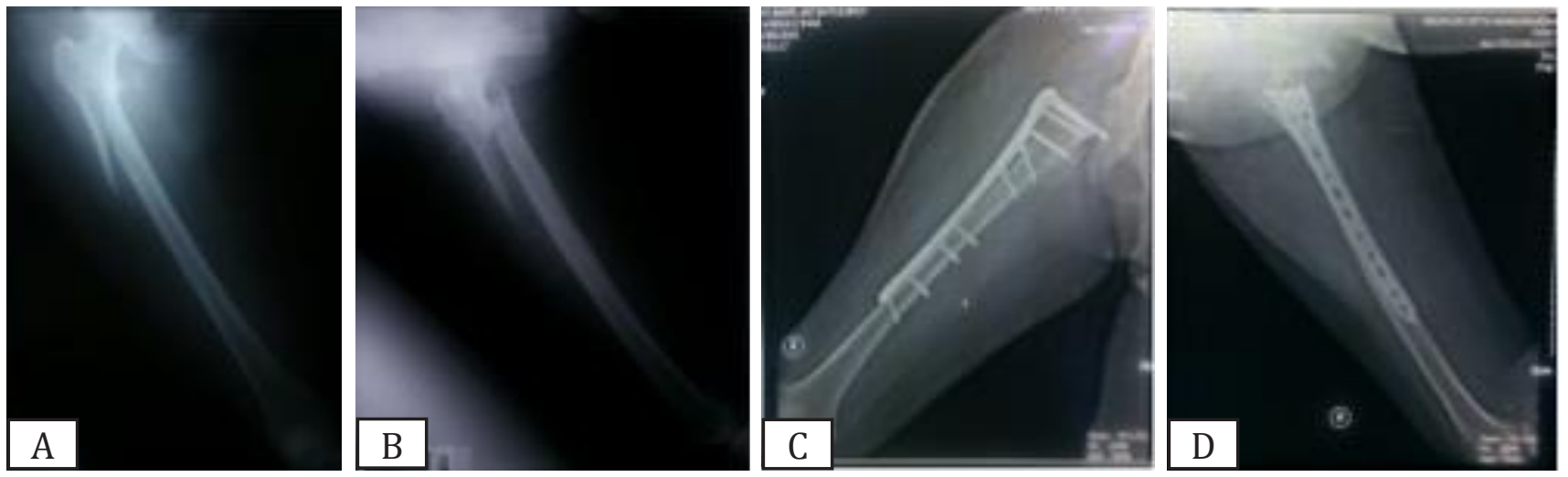

Figure 6. (A,B) Case 4. Pre-operative X-rays; (C,D) Six-month post-operative radiograph

the systemic conditions and preparation for the operation. Thus, no definitive surgery was performed less than $48 \mathrm{~h}$ after the initial injury. While waiting for the definitive surgery, the patients experienced prolonged immobilization. One neglected case patient waited for more than six months. Pre-operative problems including the timing of definitive surgery, accompanying injuries, long immobilization, general conditions, such as a history of shock, will affect the healing process of the bone and soft tissues.

In the pre-operative local status, the general condition of the bones and fractures ranged from complex fractures (floating hip) to neglected cases. However, patients with bone stock initially presented good conditions. No pathological fracture nor poor bone quality was observed, except in the third case of neglected history, where osteoporotic bone quality was affected by mild disuse of the limb Despite the intra-articular problems, in the case of floating hip (fracture of the acetabulum), femur joint conditions in this report (knee and hip) experienced no intra-articular fracture. Preoperative soft tissue conditions in this series of cases included patients without a history of open fractures and good nutritional status before the accident and without serious comorbidities. Patients with a history of smoking were also included.

Operations were carried out in RSCM with the same equipment and operator. The used implants were LDP-DF from Syntess titanium $4.5 / 5.0$ with a length of 9 or 11 holes. The screws used were a mixture of locking and non-locking screws of the same brand. Lateral femoral incision was made either in the open or LISS technique. In patients with multiple fractures, definitive surgery was performed simultaneously, causing difficulty in assessing the duration of the operation, the amount of blood loss, use of the C-arm, and other factors specific to the intraoperative management 
of femur fractures alone. In the neglected case, the added cancerous bone graft was obtained from the pelvic bone. In the case of this report, the proximal femur from the intertrochanter until the head of the femur was intact. Thus, the mounting screw in the proximal fragment can be filled with up to five implant pieces. To adjust the contour of the distal fragment fracture, locking screws were used; no significant intraoperative complications were observed.

From the results of post-operative $\mathrm{x}$-rays (Figure 5), we can conclude that the mounting screw in the region of the proximal femur presented no difficulties. Screws on the correct paths are inside the bone. A number of holes occupied alternative locations without disturbing the mounting screw joints. The sagittal arch of the distal femoral plate works gentler than the conventional plate proximal femur, eases mounting screw, ensures screw alignment, can be mounted long reach column, and avoid overlaps between the screws. The screw holes are located on the neck plate, where the plate axial curvature is the most critical. Consideration of the mounting screw in the hole depends on the fracture fragments and the quality of bone itself. With the LCP-DF made of titanium, implant failure due to fatigue critical area rarely occurred.

A few days after the surgery, several patients required admission to the Intensive Care Unit (ICU) because of the long operation duration. This condition leads to long immobilization treatment complications, such as ulcers and muscle atrophy. In the case of three patients who underwent postoperative period on schedule, early mobilization, rehabilitation exercises, and taking care of physioterapy were achieved without complications.

The mean HHS at 0,6 , and 12 months increased dramatically until reaching category at the end of the $12^{\text {th }}$ month. Average HHS increased from 22 to 75 then to 86 . Mean radiological grafting results were obtained at 6-12 months. No complications of failed implants and pull-out nor loosening of screws were observed in the patients.

Comparison of the advantages and disadvantages of each implant, including reversed LCP-DF, with other methods needs to be further investigated in terms of operating procedures, radiological and clinical outcomes, and long-term follow-up.
In conclusion, the use of reversed LCPDF in fractures of the proximal femur may be considered as alternatives in the complicated proximal femur.

\section{Conflict of interest}

The authors affirm no conflict of interest in this study.

\section{REFERENCES}

1. Baumgaertner MR, Oetgen ME. Trochanteric Hip Fractures. In: Browner BD. Skeletal Trauma: basic science, management, and reconstruction. Philadelphia: Sanders; 2003.

2. Hoffmann R, Han NP. Femur: Proximal. In: Ruedl TP, Murphy WM. AO Principles of Fracture Management. New York: Thieme; 2000. p. 441-5.

3. Beaty JH, Austin SM, Warner WC, Canale ST, Nichols L. Interlocking intramedullary nailing of femoralshaft fractures in adolescents: preliminary results and complications. J Pediatr Orthop Relat Res. 1994;14(2):178-83.

4. Wolfgang GL, Bryant MH, O'Neill JP. Treatment of intertrochanteric fracture of the femur using sliding screw plate fixation. Clin Orthop. 1982;(163):148-58.

5. Bain GI, Zacest AC, Paterson DC, Middleton J, Pohl AP. Abduction strength following intramedullary nailing of the femur. J Orthop Trauma. 1997;11(2):93-7.

6. Dodenhoff RM, Dainton JN, Hutchins PM. Proximal thigh pain after femoral nailing: causes and treatment. J Bone Joint Surg Br. 1997;79(5):738-41.

7. Dora C, Leunig M, Beck M, Rothenfluh D, Ganz R. Entry point soft tissue damage in antegrade femoral nailing: a cadaver study. J Orthop Trauma. 2001;15(7):488-93.

8. Zhang CQ, Sun Y, Jin DX, Yao C, Chen SB, Zeng BF. Reverse LISS plating for intertrochanteric hip fractures in elderly patients. BMC Musculoskelet Disord. 2010;11:166.

9. Haidukewych GJ, Ricci W. Locked plating in orthopaedic trauma: a clinical update. J Am Acad Orthop Surg. 2008;16(6):347-55.

10. Sidhom SA, Pinder R, Shaw DL. Reverse LISS plate stabilisation of a subtrochanteric fracture of the femur in a patient with osteopetrosis: is this the answer? Injury Extra. 2006;37:113-5.

11. Ma CH, Tu YK, Yu SW, Yen CY, Yeh JH, Wu CH. Reverse LISS plates for unstable proximal femoral fractures. Injury. 2010;41(8):827-33.

12. Acklin YP, Bereiter H, Sommer C. Reversed LISS-DF in selected cases of complex proximal femur fractures. Injury. 2010;41(4):427-9.

13. Ehlinger M, Brinkert D, Besse J, Adam P, Arlettaz Y, Bonnomet F. Reversed anatomic distal femur locking plate for periprosthetic hip fracture fixation. Orthop Traumatol Surg Res. 2011;97(5):560-4.

14. Haq RU, Manhas V, Pankaj A, Srivastava A, Dhammi IK, Jain AK. Proximal femoral nails compared with reverse distal femoral locking plates in intertrochanteric fractures with a compromised lateral wall; a randomised controlled trial. Int Orthop. 2014;38(7):1443-9. 